\title{
In situ lift-off InAs quantum dots by pulsed laser irradiation
}

\author{
Changwei Deng, ${ }^{1,2, a)}$ Zhenwu Shi, ${ }^{1,2, b)}$ Linyun Yang, ${ }^{1,2, a)}$ Wei Zhang, ${ }^{3}$ \\ Chen Chen, ${ }^{1,2}$ Lili Miao, ${ }^{1,2}$ Xinning Yang, ${ }^{1,2}$ Chinhua Wang, ${ }^{1,2}$ Linsen \\ Chen, ${ }^{1}$ and Changsi Peng ${ }^{1,2,4, b)}$
}

\author{
${ }^{1}$ School of Optoelectronic Science and Engineering \& Collaborative Innovation Center \\ of Suzhou Nano Science and Technology, Soochow University, Suzhou 215006, China. \\ ${ }^{2}$ Key Lab of Advanced Optical Manufacturing Technologies of Jiangsu Province \& Key \\ Lab of Modern Optical Technologies of Education Ministry of China, Soochow \\ University, Suzhou 215006, China. \\ ${ }^{3}$ Suzhou Institute of Nano-Tech and Nano-Bionics, Chinese Academy of Sciences, \\ Suzhou 215123, China. \\ ${ }^{4}$ Institute for Research in Applicable Computing, University of Bedfordshire, Park \\ Square, Luton LU1 3JU, UK.
}

InAs/GaAs quantum dots (QDs) grown by molecular beam epitaxy were subjected to in situ irradiation using a mono-beam pulsed laser. The evolution of the QD morphology was investigated as a function of irradiation intensity at temperatures of $525^{\circ} \mathrm{C}$ and $480{ }^{\circ} \mathrm{C}$. The temperature was found to exert a considerable influence on the reaction of the QDs to the irradiation. At the higher temperature $\left(525^{\circ} \mathrm{C}\right)$, both the height and width of the InAs QDs gradually decreased with increasing irradiation intensity, which was ascribed to the dominant effect of the laser desorption of indium. In contrast, at the lower temperature $\left(480{ }^{\circ} \mathrm{C}\right)$, the height of the InAs islands decreased with increasing irradiation intensity while the width exhibited unexpected broadening, which was attributed to a combination of laser desorption and laser diffusion of indium. Remarkably, at the higher temperature, laser irradiation above a certain threshold 
a) Co-first authors: These authors contributed equally to this work.

b) Electronic mail: zwshi@suda.edu.cn (Z. Shi), changsipeng@suda.edu.cn (C. Peng).

intensity resulted in the lift off of the InAs QDs to afford a clear, smooth, and perfect GaAs surface. Through subsequent growth of QDs on this surface, it was found that the QDs exhibited the same nucleation properties and optical quality as the common Stranski-Krastanov mode on an as-prepared GaAs surface. Therefore, we have developed a technology for the damage-resistant fabrication of QDs using in situ pulsed laser irradiation, which is expected to find potential applications in the manufacture of patterned QDs upon upgrading the mono-beam irradiation to multi-beam interference irradiation in the future.

Due to 3-dimentional carrier comfinement, ${ }^{1}$ semiconductor quantum dots (QDs) are used to realize a tremendous variety of applications ranging from electronic devices to photonic devices. ${ }^{2,3}$ The Stranski-Krastanov (SK) growth mode is typically used to prepare semiconductor QDs with the advantages of low defect formation, simplicity, and maturity. ${ }^{4}$ One prototypical case is the SK growth of InAs/GaAs QDs by molecular beam epitaxy (MBE), and its related devices such as photodetectors, ${ }^{5,6}$ lasers, ${ }^{7,8}$ LEDs, ${ }^{9}$ solar cells, ${ }^{10}$ etc., ${ }^{11,12}$ have been extensively fabricated. However, the cutting-edge applications based on QDs, such as single-photon emitters, ${ }^{13,14}$ quantum computation, ${ }^{15}$ and photonic crystals ${ }^{16}$ require more controllability during QD fabrication, especially with respect to site control. Since SK growth is a thermodynamic process, QD nucleation occurs randomly. This drawback has substantially limited the use of normal SK growth, and massive research attention has thus been devoted to pursuing new methods for achieving an ordered QD arrangement. For example, it has been reported that the growth of multiple layers of QDs can improve the QD arrangement by strain correlation, ${ }^{17-19}$ but this arrangement is relatively too poor in the control precision and moreover, such bottom multilayers are an undesirable parasitic structure for device applications. At present, self-assembling QDs on a pre-patterned substrateError! 
Reference source not found. is widely acknowledged as the most scalable and feasible approach for ordered QD growth. However, there are still several shortcomings greatly challenged the technology of patterned substrate: (a) Oxidation, pollution, and crystal damage of the substrate inevitably occur during the patterning process; (b) It is very hard to achieve devices with two or more patterned QD layers especially whose interspace is quite small, because the first buried QD layer may be damaged when performing the second patterning step on the interlayer.

In this paper, we investigated an in situ pulsed laser irradiation (LIR) of InAs QDs grown on a GaAs (001) surface. It was found the LIR could targeted lift off the InAs QDs from the substrate to leave a clear, smooth, and perfect GaAs surface. Therefore, a potential damage-resistant fabrication of patterned QD can be easily foreseen and realized by upgrading the mono-beam irradiation to multi-beam interference irradiation that could solve all of the aforementioned problems of the patterned substrate approach. First, pollution and oxidation are directly eliminated in the in-situ process and meanwhile it is also proven non-destructive. Second, if the strain correlation between the QD multilayers, ${ }^{18-23}$ is neglected, our technique can freely fabricate complex QD structures via layer-by-layer stacking of the patterned QD layers in a similar manner to 3D printing with considerable cost and time savings.

The experiments were performed on a special MBE system equipped with a laser viewport for performing in situ LIR. First, a $500 \mathrm{~nm}$ GaAs buffer layer was deposited on a quarter of 2-inch deoxidized GaAs (001) substrate at $600{ }^{\circ} \mathrm{C}$. Then, the temperature was separately reduced to $525^{\circ} \mathrm{C}$ (samples A) and $480{ }^{\circ} \mathrm{C}$ (sample B) for the growth of InAs QDs, for which the growth rate, deposition thickness, and As flux were 0.011 $\mathrm{ML} / \mathrm{s}, 1.7 \mathrm{ML}$, and $8.0 \times 10^{-7}$ Torr, respectively. Then the centers of the two samples were immediately followed by a mono-beam LIR with a single pulse (wavelength: 355 $\mathrm{nm}$, duration: $10 \mathrm{~ns}$, energy: $10 \mathrm{~mJ}$ ). The entire process was monitored by reflection high energy electron diffraction (RHEED). After irradiation, the surface morphology of the two samples were examined by tapping-mode atomic force microscopy (AFM). Because the laser spot is much smaller than the substrate, both the non-irradiated region (NIRR) and irradiated region (IRR) could be examined simultaneously. 
Figure 1(a) shows the observed morphology of the NIRR for sample A $\left(525^{\circ} \mathrm{C}\right)$; the QD growth is just at the critical onset of nucleation with a very broad distribution as shown in Figs. 1(e) and (h). Fig. 1(d) shows the morphology of the irradiated region center (IRRC), where the InAs QDs have completely disappeared to afford a flat and clear surface with an obvious step flow (white arrows). The RHEED revealed that the LIR immediately caused the dot-like pattern to abruptly switch to a bright streak-like diffraction pattern of a pure GaAs surface. This result convincingly demonstrates that LIR can cleanly lift off the InAs QDs from the substrate to leave a clear, smooth, and perfect GaAs surface. To further clarify the LIR, we scanned the transition between the NIRR and the IRR and two representative regions (denoted IRR1 and IRR2 with

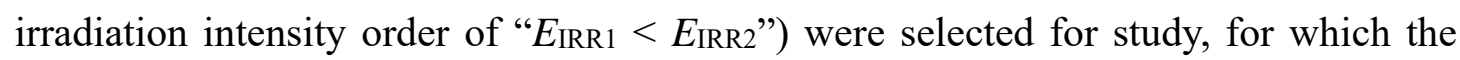
results are presented in Figs. 1(b) and (c), respectively. Figs. 1(f,g) and (i,j) show the corresponding width and height distributions of the InAs QDs in IRR1 and IRR2. With irradiation intensity increasing, the InAs QDs gradually decreased in both width (Figs. 1(e-g)) and height (Figs. 1(h-j)) and ultimately disappeared. While for sample B $\left(480{ }^{\circ} \mathrm{C}\right)$, four regions denoted NIRR, IRR1, IRR2, and IRRC were again selected and investigated (Figs. 2(a-d)). In the IRRC (Fig. 2(d)), the InAs QDs were removed but the remained surface was very rough. Thus from RHEED, the observed GaAs reconstruction streaks were dispersed and not bright. In contrast to sample A, it appears that the surface step flows were cracked into many irregular atomic fragments in sample $\mathrm{B}$ (white arrows). The evolution of the InAs islands with the irradiation intensity is also remarkable; as shown in Figs. $2(\mathrm{e}-\mathrm{j})$, the height of the InAs islands gradually decreased in a similar manner to sample A, but the width abnormally broadened. Consequently, the substrate temperature exerts a strong influence on the effect of LIR on InAs QDs. 


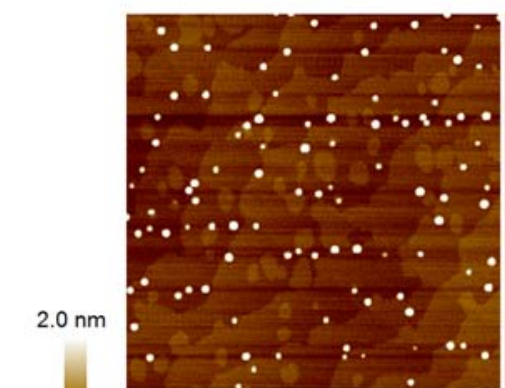

(a) NIRR

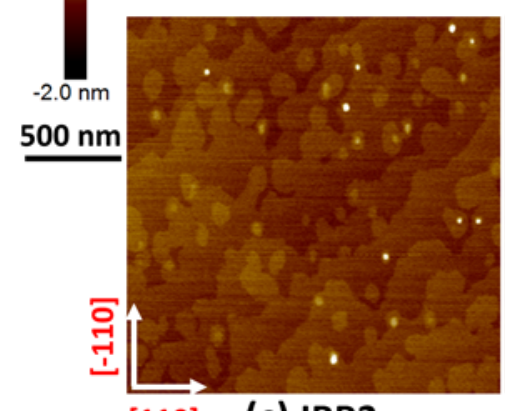

[110] (c) IRR2

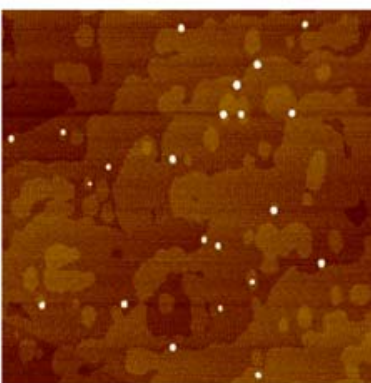

(b) IRR1

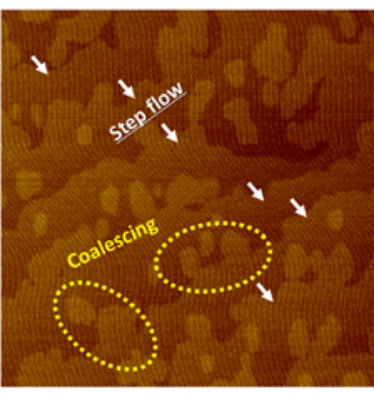

(d) IRRC
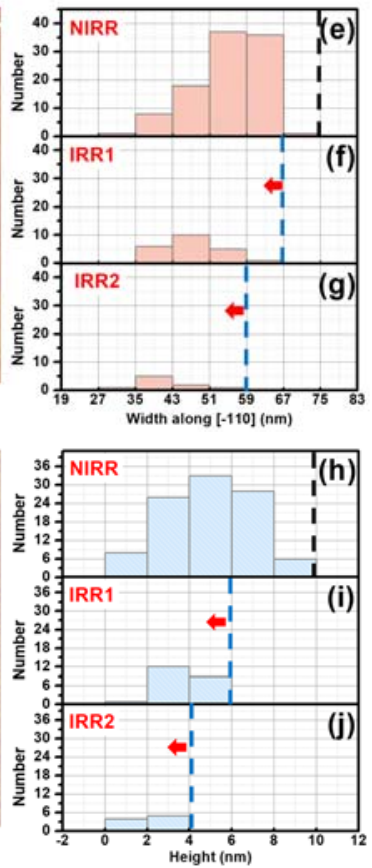

FIG. 1. (a-d) AFM morphology results in the (a) NIRR, (b) IRR1, (c) IRR2, and (d) IRRC of sample $A$, and $(e-j)$ corresponding histograms of $(e-g)$ width and $(h-j)$ height distributions for the InAs QDs in NIRR, IRR1, and IRR2. 


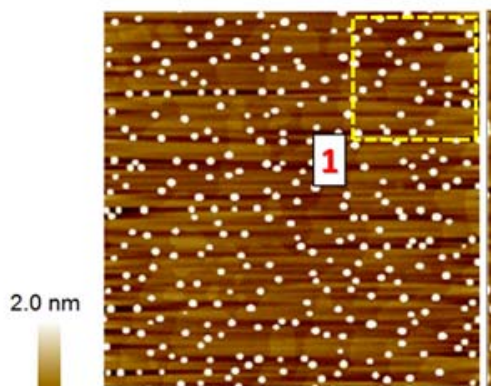

(a) NIRR

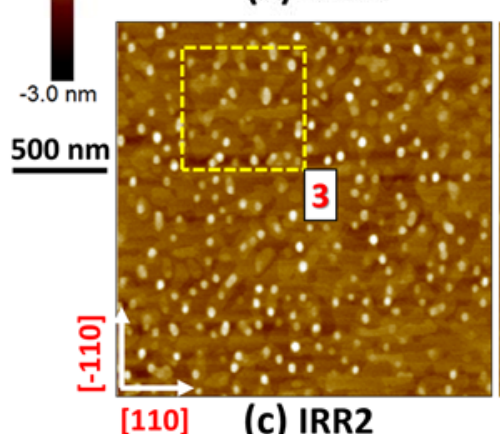

(c) IRR2

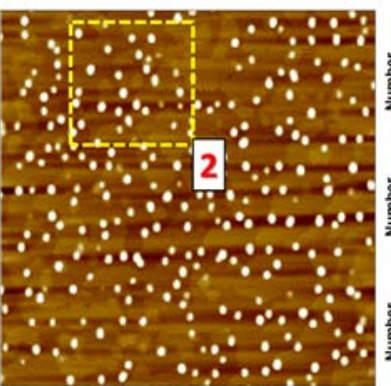

(b) IRR1

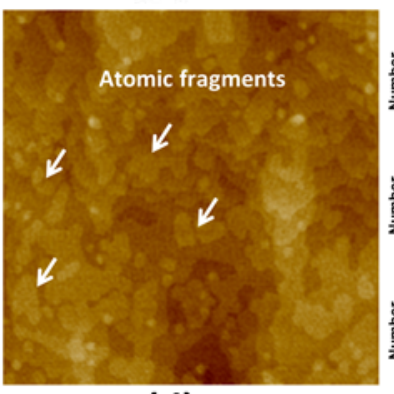

(d) IRRC

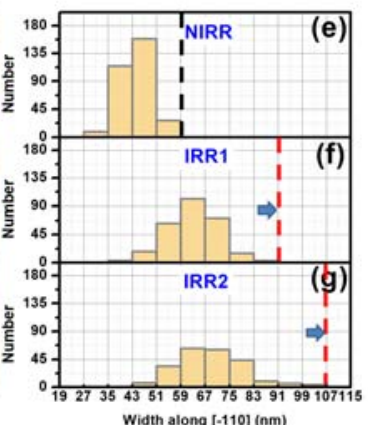

Width along $[-110](\mathrm{nm})$

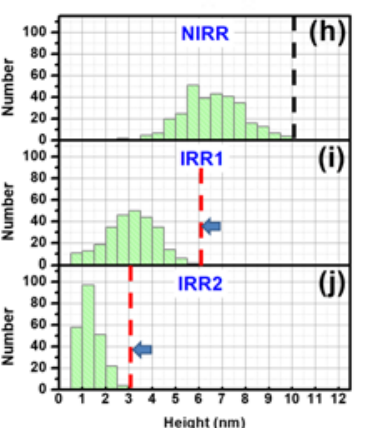

Height $(\mathrm{nm})$

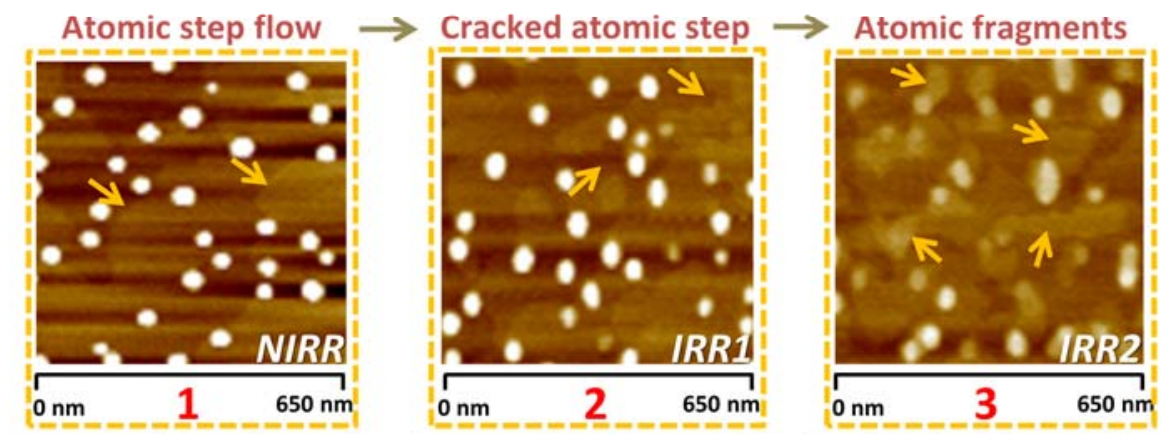

Evolution of the background surface with irradiation intensity

FIG. 2. (a-d) AFM morphology results in the (a) NIRR, (b) IRR1, (c) IRR2, and (d) IRRC of sample B, and $(e-j)$ corresponding histograms of $(e-g)$ width and $(h-j)$ height distributions for the InAs QDs in NIRR, IRR1, and IRR2. At the bottom, three enlargements of yellow dashed squares (denoted 1-3) in (a-c) are presented. 
To directly visualize the differences, we selected one typical island from the NIRR, IRR1, and IRR2 of each sample, for which the section profiles are presented in Figs. 3(a) and (b). It clearly demonstrates that, for sample A, the body of the InAs QD consistently maintained a "dot" shape and shrunk step-by-step during the LIR. In contrast, during the LIR of sample B, the InAs QD body did not exhibit a "dot" shape but deformed into 2D islands. To interpret these interesting phenomena, it should first be pointed out that the InAs QDs grown at $525^{\circ} \mathrm{C}$ (Fig. 1(a)) possessed a lower density and smaller size than those grown at $480{ }^{\circ} \mathrm{C}$ (Fig. 2(a)), despite the identical InAs deposition amount. This reflects that the adsorption coefficient of indium sharply decreased at $525{ }^{\circ} \mathrm{C}$; in other words, the indium atoms in the QDs will also become extremely active and easy to evaporate. Consequently, upon LIR of sample A, the indium atoms will easily be excited by the laser to overcome the vacuum barrier (Fig. 3(c), as indicated by the red arrow) and desorb from the surface. The laser-induced desorption of indium is depicted in Fig. 3(d): the outer indium atoms would be expected to desorb prior to those inside the QD body, which explains the gradual shrinkage of the QDs (indicated by black arrows) with increasing irradiation intensity. Moreover, the InAs QDs with a relatively small size (as observed in Fig. 1(a), a large proportion of the QDs possessed a small size) would also be completely desorbed immediately after irradiation thus leads to a rapid QD density reduction in IRR1 (Fig. 1(b)). Finally, the clear and smooth GaAs surface observed in the IRRC (Fig. 1(d)) can be simply explained by the target desorption of indium owing to the intrinsic difference in bond strength between InAs and GaAs (see the vacuum barrier gap between In and $\mathrm{Ga}$, as depicted in Fig. 3(c)). In contrast, for sample B, the indium will become more stable at $480{ }^{\circ} \mathrm{C}$ and thus the desorption probability may be relatively depressed under the same irradiation intensity. Consequently, some of the indium atoms will fail to desorb from the surface, but they might overcome the lower diffusion barrier (the black arrow in Fig. 3(c)) to migrate on the surface. As shown in Fig. 3(e), we therefore propose a combination of laser desorption (red arrows) and diffusion (green arrows) to explain the observed abnormal width extension in sample B. When referring to the issue of surface diffusion, it is worth mentioning that the diffusion barrier along the [-110] 


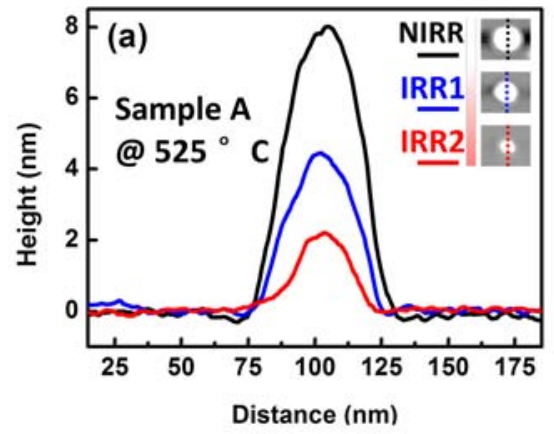

(c)

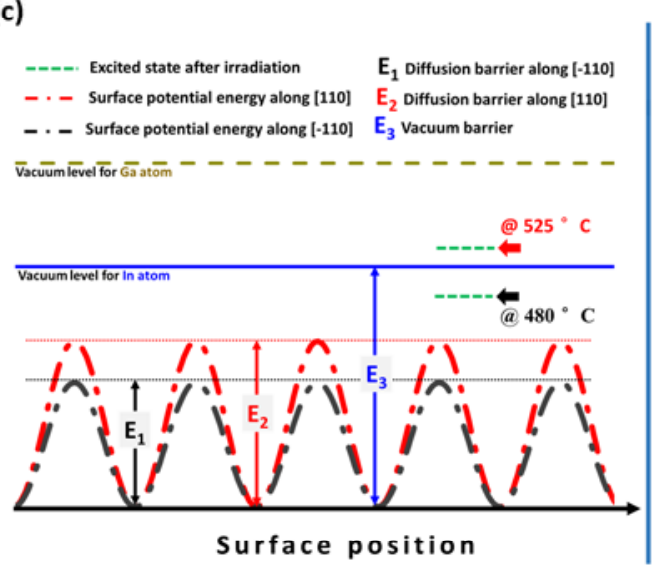

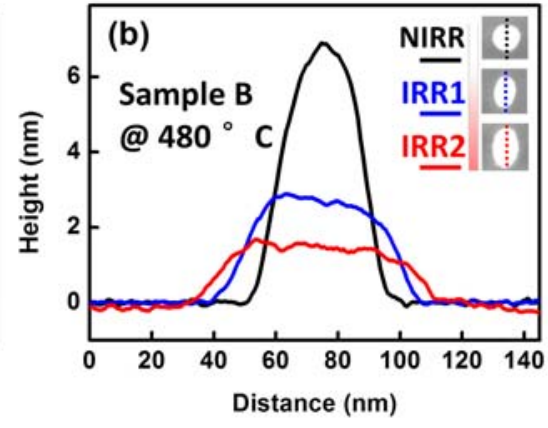

Laser

desorption

In

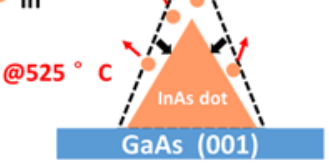

(e)
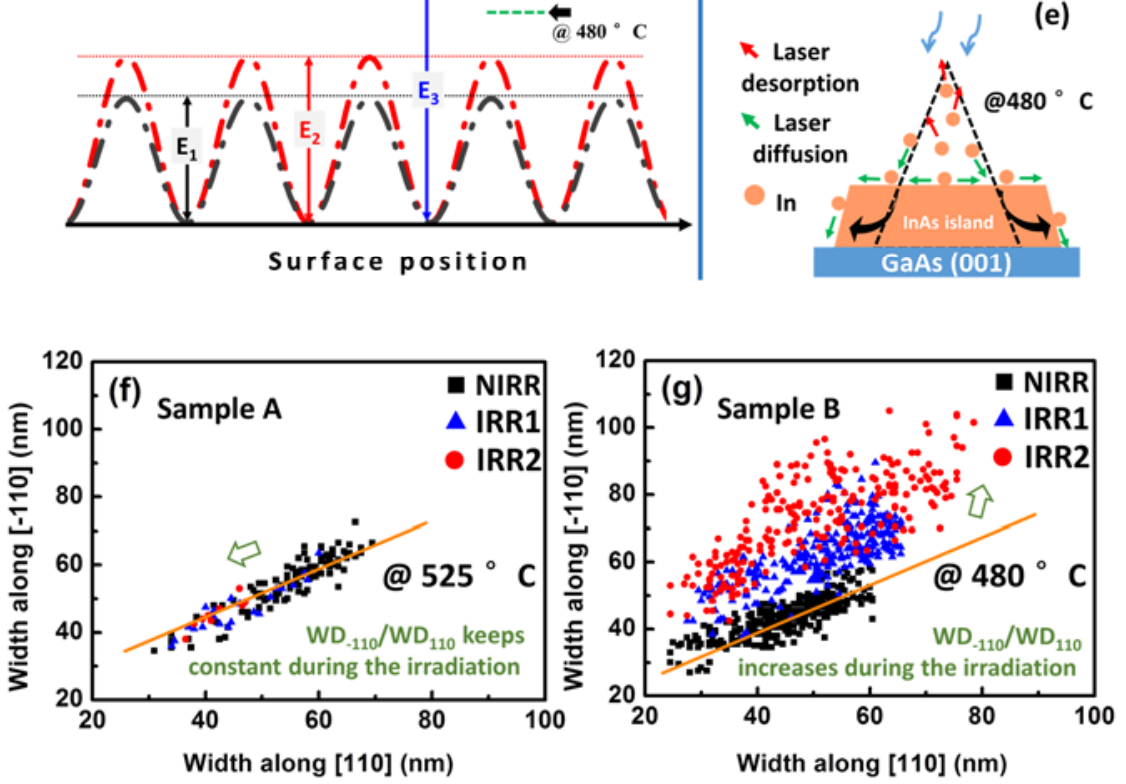

FIG. 3. (a,b) Section profiles of three typical InAs islands selected from the NIRR, IRR1, and IRR2 of (a) sample A and (b) sample B. (c) Possible excited state levels (after irradiation) for In atoms at $525^{\circ} \mathrm{C}$ and $480{ }^{\circ} \mathrm{C}$, and $(\mathrm{d}, \mathrm{e})$ the corresponding reactions of indium atoms to irradiation at (d) $525^{\circ} \mathrm{C}$ and (e) $480{ }^{\circ} \mathrm{C}$. (f,g) Relationships between the widths along the [-110] and [110] directions of the InAs islands in the NIRR, IRR1, and IRR2 of (f) sample A and (g) sample B.

144 direction is smaller than that along the [110]direction for $\mathrm{In} / \mathrm{GaAs}^{24,25}$ which usually 145 forms non-symmetrical surface morphology. Therefore, we measured the widths along 146 the [-110] (WD-110) and [110] (WD 110$)$ directions for all of the InAs islands shown in 
Figs. 2(a-c), and the results are presented in Fig. 3(g). It can be clearly seen that the InAs islands were elongated along the [-110] direction with an increasing WD. 110/WD 110 ratio after irradiation. For comparison, Fig. 3(f) shows the corresponding data for sample A, in which all of the InAs QDs maintained a consistent WD-110/WD110 ratio during irradiation. In addition, the only remaining point to be clarified is why the surface shown in Fig. 2(d) is so rough. It is well known that the InAs QDs grow on wetting layers that are rich in indium. When these indium atoms are evaporated, the original surface atomic layer will randomly crack into small fragments. When the substrate temperature is not sufficiently high to provide enough surface diffusion, these fragments would be unable to coalesce back in time. To test this hypothesis, we closely examined three enlargements of yellow dashed squares (denoted 1-3) in Figs. 2 (a-c) and clearly observed an evolution from "atomic step flow" to "atomic fragments" on the background surface (as indicated at the bottom of the graphic). In contrast, the background surface of sample A remained almost unchanged during LIR, which was ascribed to the more efficient coalescencing (yellow dashed circles in Fig. 1(d)) at higher temperature of $525^{\circ} \mathrm{C}$.

Taken together, the results from sample A indicate that the surface shown in Fig. 1(d) should has a very low concentration of defects. To thoroughly evaluate the surface quality, we prepared sample $\mathrm{C}$ by depositing $1.8 \mathrm{ML}$ of $\mathrm{InAs}$ at $525^{\circ} \mathrm{C}$ to fully form the QDs, subjected it to in situ LIR, and finally capped it with another 1.8 ML of InAs. AFM and photoluminescence measurements were then conducted. The AFM morphologies of the IRRC and NIRR of sample C are presented in Figs. 4(a) and (b). For comparison, sample D with only a single layer of $1.8 \mathrm{ML}$ InAs deposited on the GaAs substrate was also prepared and the AFM results are presented in Fig. 4(c). The IRRC of sample $\mathrm{C}$ exhibited almost the same QD morphology as sample D that means the LIR cleanly lift off the first 1.8 ML InAs QD layer. While for the NIRR (Fig. 4(b)), where the actual total deposition amount is 3.6 ML, both the QD density and size became much larger even creating some huge InAs islands (red arrows). Furthermore, the photoluminescence spectrum (Fig. 4(d)) revealed that the optical quality of the InAs QDs in the IRRC was also as excellent as that of sample D, without any degradation. 
Therefore, in situ LIR is proven to be a promising technique for the fabrication of damage-resistant QDs.

Finally, considering that the growth temperature may also affect the wetting and strain and thus afford QDs with different physicochemical properties, in order to figure out whether the growth temperature also played an important role for the morphological differences between sample A and B which are respectively grown at different temperature. It would make more sense to comparing the dots grown at the same temperature and then change the temperature before irradiation, so we prepared a final sample (sample E) in which the QDs were first grown at $480{ }^{\circ} \mathrm{C}$ and then irradiated at $525^{\circ} \mathrm{C}$. As presented in Fig. 4(e), the evolution of the QD morphology with increasing irradiation intensity behaved the same as sample A. It reflects "in situ lift-off of InAs" predominantly depends on the irradiation temperature and intensity, in other words, the LIR should be applicable to lift off any InAs QD regardless of its exact preparation method and morphology.

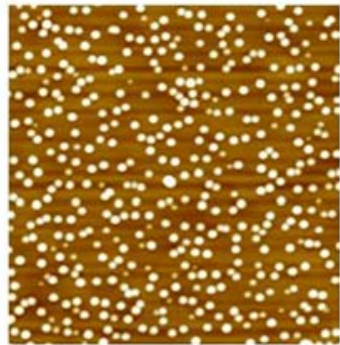

Sample C - IRRC

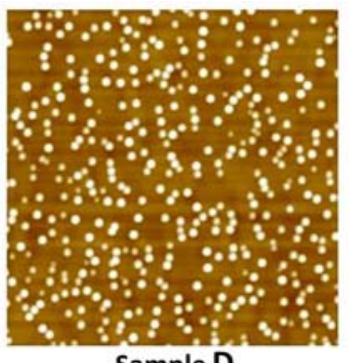

Sample D

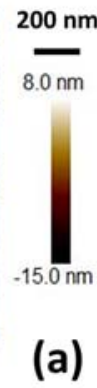

(a)

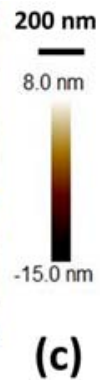

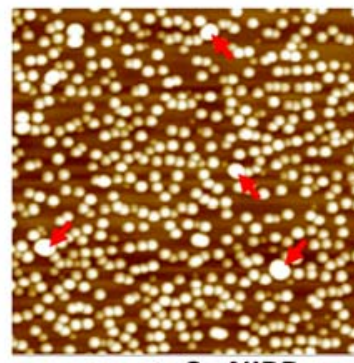

Sample C - NIRR

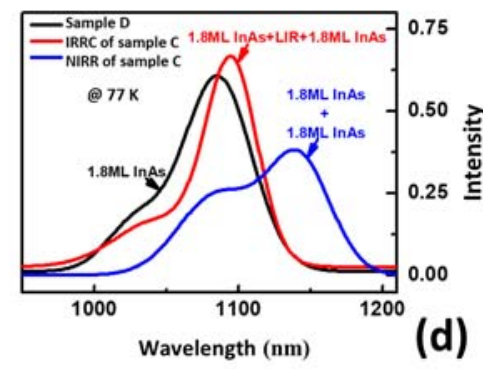

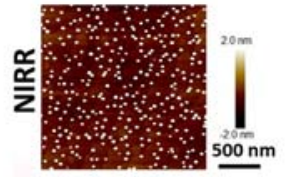

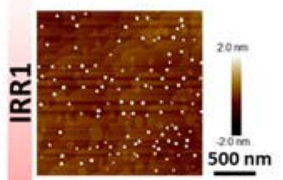

(b)
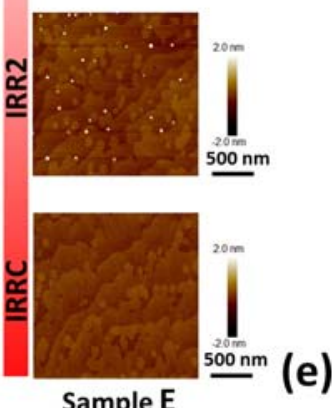

FIG. 4. (a-c) AFM morphology results of the InAs QDs in the (a) IRRC of sample C and (b) NIRR of sample C and (c) sample D, and (d) their corresponding photoluminescence spectra. (e) Evolution of the QD morphology with increasing irradiation intensity for sample E, where the QDs were grown at $480{ }^{\circ} \mathrm{C}$ and then irradiated at $525^{\circ} \mathrm{C}$. 
In summary, we have investigated the in situ LIR of InAs QDs grown on a GaAs substrate by MBE. For the sample irradiated at the lower temperature $\left(480{ }^{\circ} \mathrm{C}\right)$, the irradiation of the InAs QDs was found to be governed by a combination of laser-induced desorption and diffusion of indium; while for the sample irradiated at the higher temperature $\left(525^{\circ} \mathrm{C}\right)$, the irradiation process was dominated by laser desorption only. At both temperatures, LIR was found to induce complete lift off of the InAs QDs from the substrate. Especially for the case of $525{ }^{\circ} \mathrm{C}$, the photoluminescence spectroscopy demonstrated that QDs subsequently grown on the irradiated surface after the LIR have exhibited the same optical quality as the common SK growth mode on an as-prepared GaAs surface. Therefore, we have developed a defect-free technology for QD fabrication by using in situ pulsed laser irradiation to lift off InAs QDs from the GaAs surface and this technology is compatible with the common epitaxy equipment with avoiding pollution and oxidation as well. In future work, we expect to facilely develop this method for the patterned lift-off of QDs by upgrading the mono-beam irradiation to multi-beam interference irradiation.

The research was supported by the National Natural Science Foundation of China (NSFC, Grant No. 11504251), the Priority Academic Program Development of Jiangsu Higher Education Institutions (PAPD), the International Cooperation Project (Grant No. 2014DFG12600) by MOST, and the Natural Science Research Project of Jiangsu Higher Education (Grant No. 12KJA140001).

\footnotetext{
${ }^{1}$ K. A. Fischer, L. Hanschke, J. Wierzbowski, T. Simmet, C. Dory, J. J. Finley, J. Vučković, and K. Müller, Nature Physics 13, 649 (2017).

${ }^{2}$ B. S. Mashford, M. Stevenson, Z. Popovic, C. Hamilton, Z. Zhou, C. Breen, J. Steckel, V. Bulovic, M. Bawendi, S. Coe-Sullivan, and P. T. Kazlas, Nature Photonics 7, 407 (2013).

${ }^{3}$ M. Davanco, J. Liu, L. Sapienza, C.-Z. Zhang, J. Vinícius De Miranda Cardoso, V. Verma, R. Mirin, S. W. Nam, L. Liu, and K. Srinivasan, Nature Communications 8, 889 (2017).
} 
${ }^{4}$ B. H. Kim, M. S. Onses, J. B. Lim, S. Nam, N. Oh, H. Kim, K. J. Yu, J. W. Lee, J.-H. Kim, S.-K. Kang, C. H. Lee, J. Lee, J. H. Shin, N. H. Kim, C. Leal, M. Shim, and J. A. Rogers, Nano Letters 15, 969 (2015).

${ }^{5}$ D. Pan, E. Towe, and S. Kennerly, Applied Physics Letters 73, 1937 (1998).

${ }^{6}$ J. Wu, Q. Jiang, S. Chen, M. Tang, Y. I. Mazur, Y. Maidaniuk, M. Benamara, M. P. Semtsiv, W. T. Masselink, K. A. Sablon, G. J. Salamo, and H. Liu, ACS Photonics 3, 749 (2016).

${ }^{7}$ J. Y. Yan, Q. Gong, C. Z. Kang, H. X. Xu, C. F. Cao, Y. Y. Li, S. M. Wang, and H. L. Wang, Journal of Crystal Growth 425, 373 (2015).

${ }^{8}$ B. Lingnau, K. Lüdge, B. Herzog, M. Kolarczik, Y. Kaptan, U. Woggon, and N. Owschimikow, Physical Review B 94, 014305 (2016).

${ }^{9}$ J. P. Lee, E. Murray, A. J. Bennett, D. J. P. Ellis, C. Dangel, I. Farrer, P. Spencer, D. A. Ritchie, and A. J. Shields, Applied Physics Letters 110, 071102 (2017).

${ }^{10}$ S. Suraprapapich, S. Thainoi, S. Kanjanachuchai, and S. Panyakeow, Solar Energy Materials and Solar Cells 90, 2968 (2006).

${ }^{11}$ C. E. Dimas, H. S. Djie, and B. S. Ooi, Journal of Crystal Growth 288, 153 (2006).

${ }^{12}$ C. Y. Ngo, S. F. Yoon, W. K. Loke, Q. Cao, D. R. Lim, V. Wong, Y. K. Sim, and S. J. Chua, Applied Physics Letters 94, 143108 (2009).

${ }^{13}$ A. Imamoglu, D. D. Awschalom, G. Burkard, D. P. DiVincenzo, D. Loss, M. Sherwin, and A. Small, Physical Review Letters 83, 4204 (1999).

${ }^{14}$ E. Waks, K. Inoue, C. Santori, D. Fattal, J. Vuckovic, G. S. Solomon, and Y. Yamamoto, Nature 420, 762 (2002).

15 T. F. Watson, S. G. J. Philips, E. Kawakami, D. R. Ward, P. Scarlino, M. Veldhorst, D. E. Savage, 

555, 633 (2018).

${ }^{17}$ H. Z. Song, T. Usuki, T. Ohshima, Y. Sakuma, M. Kawabe, Y. Okada, K. Takemoto, T. Miyazawa, 1099 (1985).

${ }^{22}$ N. N. Ledentsov, V. A. Shchukin, M. Grundmann, N. Kirstaedter, J. Böhrer, O. Schmidt, D. Heydenreich, Physical Review B 54, 8743 (1996). Alferov, Applied Physics Letters 72, 942 (1998). 
